\section{Dr Maclean}

My own view is that any organisation which knowingly relies on another agency to provide the new technology on which its manufacturing future is going to depend deserves everything that comes to it. I admit that the large chemical companies and fibre producers perhaps encouraged this approach; after all, a customer in thrall has fewer competitive options to select. Technical assistance is widely available though throughout the UK, and the alternative certainly does not mean that individual small and medium-sized dyeing and textile companies have to set up their own extensive research departments. If such companies have developed a clear technical strategy for themselves, and this must be as much a part of corporate planning as any other functional area, a plan for accessing suitable resources must axiomatically follow. Companies should be considering such questions as the following

(a) What is our total R \& D budget?

(b) How much of that must be in-house?

(c) How much can we contract out?

(d) What are the essential technical topics to be covered?

(e) Where are the sources of appropriate technical advice? (research associations, universities, suppliers, customers)

(f) How much do we contract on an exclusive basis, and how much to we take advantage of club projects?

If companies at the end of this modest check-list still feel that they cannot afford to spend on their technological future, then the new situation is probably serious beyond redemption.

\title{
Bubble size distribution and energy dissipation in foam mixers
}

\section{A B J Kroezen and J Groot Wassink}

\author{
Twente University of Technology, Enschede, The Netherlands
}

\begin{abstract}
The bubble size distribution of a foam produced in a rotor-stator mixer has been determined as a function of several mixing parameters such as the rotor speed, residence time, gas/liquid ratio and the viscosity of the liquid used. A Newton-Reynolds expression for a foam mixer has been determined using energy consumption measurements. Two types of shear fields have been distinguished in the foam mixer, laminar and turbulent, the type of shear field depending on the properties of the foam mixer. After a certain mixing time the bubble size distribution characterised by the mean bubble size was found to reach a stationary value. The stationary bubble size distribution has been correlated with the mixing conditions by a critical Weber number. Both in the laminar and in the turbulent shear field bubble size distributions have been determined as a function of several mixing parameters.
\end{abstract}

\section{INTRODUCTION}

Gas emulsions are colloids with a gas as the dispersed phase and a liquid as the continuous phase. Gas emulsions with a high fraction of gas $(>50 \%)$ are called foams, and their structure can be characterised by the blow ratio (volumetric ratio between gas and liquid) and the bubble size distribution. The structure of the foam is an important parameter that determines properties of the foam, such as its stability and rheology. In foam application technology, where foam is used as an intermediate to apply small quantities of liquid on structured materials such as textiles, nonwovens and carpets, stability and rheology are important properties. The rheology of foam changes as a function of bubble size. A foam with a smaller mean bubble diameter has a higher viscosity. The stability of the foam is also a function of the bubble size distribution. For instance, the rate of drainage is lower in a foam with a small mean bubble diameter than in foam with a larger one. The bubble size distribution may also be used as an independent parameter to change the application conditions. The way the bubble size distribution can be varied by changing, for instance, the number of revolutions per second of the mixer is therefore an important question. In our investigation the formation of the foam structure in a rotor-stator mixer has been studied. The foaming process is carried out in a mixing space in which a gas is dispersed in the liquid phase by supplying a certain amount of energy.

The foam structure produced characterises the foaming process. The first property mentioned, the blow ratio, is determined by the ratio of gas and liquid flow rates supplied to the mixer. The boundary of the working area of the foam mixing process can be defined as the maximum flow rate at which gas can be dispersed in the foam phase as a function of the liquid flow rate producing a foam without slugs, which are very large bubbles containing the unmixed gas leaving the mixer [1].

The second structure-determining property, the bubble size distribution, is on the one hand determined by mixer properties (geometry, residence time and rotor velocity) and on the other hand by the properties of the gas-liquid system (blow ratio, surface properties and viscosity). 


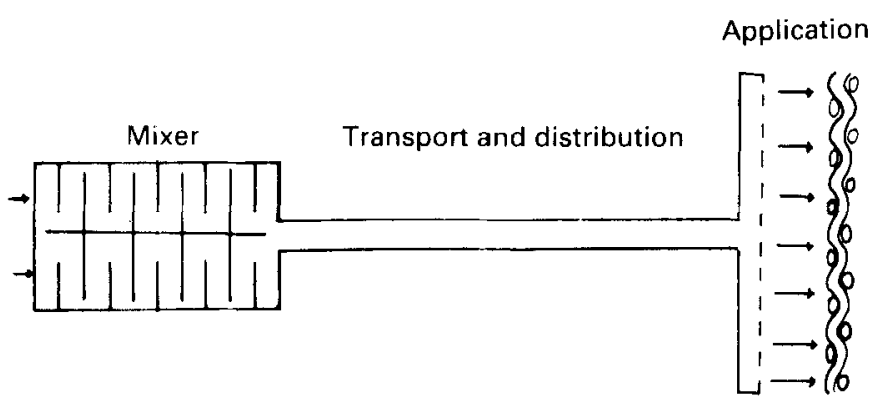

Figure 1- Foam application unit

Figure 1 schematically represents a foam application unit. One of the unit operations is the production of the foam. The incoming gas and liquid are mixed under pressure in the foam mixer and expanded from the mixer pressure, $P_{\mathrm{m}}$, to atmospheric pressure, $P_{\mathrm{o}}$, at the application point. The mixer pressure is built up by the pressure drop over the transport tube. The blow ratio, $B_{\mathrm{m}}$, of the foam in the mixer will be expanded to a blow ratio $B_{\mathrm{o}}$ at the end of the tube. The expansion of the foam in the tube can be approximated by Eqn 1 [1]:

$$
B_{\mathrm{m}}=\frac{B_{\mathrm{o}} P_{\mathrm{o}}}{P_{\mathrm{m}}}
$$

where $B_{\mathrm{o}}=F_{\mathrm{g}} / F_{\mathrm{l}}$.

The expansion of the bubbles in the foam, with a diameter $d_{m}$ in the mixer, to bubbles with diameter $d_{o}$ at the end of the tube can be approximated by Eqn 2, assuming no other mechanisms affecting bubble size act upon the dispersion:

$$
d_{\mathrm{o}}=d_{\mathrm{m}}\left(\frac{P_{\mathrm{m}}}{P_{\mathrm{o}}}\right)^{1 / 3}
$$

In the foam mixer a foam is produced with a certain bubble size distribution. The distribution changes by the expansion of the foam, as already mentioned, and by the destabilisation of the foam by means of a number of structure deformation mechanisms [2,3]. The first mechanism of destabilisation is the drainage of the liquid present in the foam via the Plateau borders due to the gravity field. This will cause inhomogeneity of the blow ratio in the foam on a larger scale. The second mechanism of deformation is diffusion of the gas from smaller bubbles with a higher internal pressure to larger bubbles through the thin lamella between the bubbles $\left(\Delta P=\gamma\left(1 / r_{1}-1 / r_{2}\right)\right)$. This change in the bubble size distribution with time is also dependent on the initial bubble size distribution $[2,4]$. The third mechanism is bursting of the lamellae. The double-layer repulsion of the two surfaces of a lamella stabilises the foam. A lamella will burst when the two surfaces make contact. Due to mechanical and thermal forces weak spots in the lamellae can be obtained, which can result in bursting of the lamellae.

\section{Stability of bubbles in several flow regimes}

\section{Laminar shear field}

The first systematic investigations in the area of deformation of emulsion drops in the laminar shear field were published by Taylor $[5,6]$. He gave two dimensionless numbers that determine the deformation of the bubbles. (Eqns 3 and 4):

$$
\begin{aligned}
& (W e)=\frac{D_{\mathrm{r}} d \mu}{\gamma} \\
& \lambda=\frac{\mu_{\mathrm{i}}}{\mu_{\mathrm{a}}}
\end{aligned}
$$

Eqn 3 gives the ratio of the shear forces working on the drop and the internal capillary forces, while Eqn 4 gives the ratio between the viscosity of the dispersed phase and the viscosity of the continuous phase. The relative deformation of the bubble (Figure 2) can be characterised by the deformation number given in Eqn 5 :

$$
(D f)=\frac{a-b}{a+b}
$$
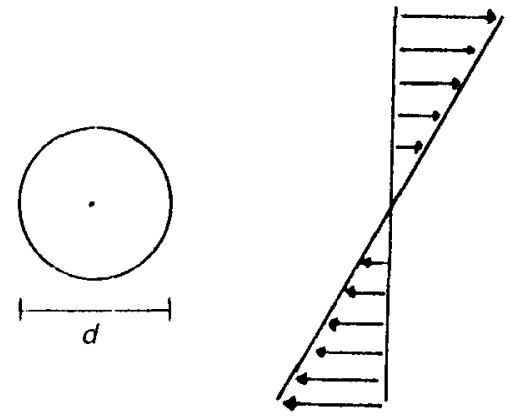

Dr

Figure 2 -Deformation of the bubble

For gas emulsions $\lambda$ is much less than unity. Rumscheid and Mason [7] measured a critical Weber number and deformation number in Couette flow $[(W e)=0.59,(D f)=0.52]$. A Weber number higher than the critical Weber number will lead to bubble splitting. Kiefer [8] has reviewed some measured critical Weber numbers over a wide range of $\lambda$ values in several types of flow.

\section{Turbulent shear field}

Several authors have proved experimentally that in the turbulent flow region the critical Weber number determines the maximum stable bubble diameter:

$$
(W e)=\frac{T d_{\max }}{\sigma}
$$

The theory of turbulence of Kolmogoroff may be used to describe the acting shear stresses [9]. The theory describes the turbulence in terms of eddies in the flow. In a turbulent field the largest eddies are of similar dimensions to those of the mixer components. These largest eddies are dependent on the shape of the mixer. The energy of the largest eddies is transferred to smaller eddies. In the smallest eddies the energy is dissipated by viscous forces. The scale of the primary eddies is $L_{1}$ and of the smallest eddies is $L_{\mathrm{s}}$. The scale of the smallest eddies can be calculated using Eqn 7:

$$
L_{s}=\mu^{3 / 4} \rho^{-1 / 2}\left(\frac{Q_{w}}{V}\right)^{-1 / 4}
$$

At a value $e$, mid-way between $L_{1}$ and $L_{\mathrm{s}}$, the flow has a so-called isotropic turbulent character. These eddies are 
independent in size and quantity of the primary eddies. The mean square fluctuating velocity over a distance $e$ is given by Eqn 8 :

$$
v^{2}=c_{1}\left(\frac{Q_{w}}{V}\right)^{2 / 3}\left(\frac{e}{\rho}\right)^{2 / 3}
$$

The shear stress $T$ that gives bubble splitting on the scale of the isotropic turbulence is given by Eqn 9 :

$$
T=v^{2} \rho
$$

The relationship for the maximum stable bubble size in the isotropic turbulent field is found by substituting Eqns 6,8 and 9 into Eqn 10 :

$$
d_{\max }=\left(\frac{(W e)_{c} \gamma}{c_{1}}\right)^{0.6}\left(\frac{Q_{w}}{V}\right)^{-0.4} \rho_{\mathrm{s}}^{-0.2}
$$

Batchelor found a value of 2 for $c_{1}[9]$

\section{Bubble size distribution functions}

The bubble size distribution of dispersions is often approximately described by statistical functions. The several statistical functions used have almost no relation to the physical background of the dispersion process. In Table 1 a number of frequently used statistical functions are represented.

\section{TABLE 1}

\section{Statistical distribution functions}

\begin{tabular}{llc}
\hline \multicolumn{1}{c}{ Distribution } & Application & Ref. \\
\hline$f(r)=\frac{2.08 r}{\left(1+0.37 r^{2}\right)^{4}}$ & Foams & {$[2]$} \\
$f(r)=(32 / \pi) r^{2} \exp \left(-4 r^{2} / \pi\right)$ & Liquid-liquid & {$[16]$} \\
$y$-distribution & Foams & {$[4]$} \\
L.og-normal distribution & Liquid-liquid & {$[17]$} \\
\hline
\end{tabular}

Population balance models with a relation to the physical background of the dispersion process are also used [10-12]. Usually two mechanism are taken into account, bubble splitting and bubble coalescence. Based on simple assumptions, analytical solutions are obtained [10] and numerical solutions can be obtained for more complex assumptions.

\section{DESCRIPTION OF EQUIPMENT}

The foam mixer used is a rotor-stator mixer, with an annular mixing space containing ten rotor discs and ten stator discs [1]. Every disc has 18 mixing pens. Gas and liquid are supplied separately to one side of the mixer and the foam produced flows into the transport tube on the other side of the mixer. Two equipment frames are used for the measurements. In the first equipment frame foam is generated in a mixer, as described previously [1]. The foam flows directly from the mixer to a camera section, where the bubble size distribution is recorded photographically through a microscope with dark field illumi- nation. The foam flows through a cell with a perspex window. Just before photographing the foam, the taps on both sides of the cells are closed. The bubble size distribution is recorded within $5 \mathrm{~s}$ after leaving the mixer The gas diffusion in the foam can change the bubble size distribution very quickly, especially in highly dispersed foams. Figure 3 shows a single rotor mixer used to measure the bubble size distribution as a function of several mixing parameters. In the single rotor mixer unit, however, a certain quantity of gas and liquid is mixed batchwise. The advantage of this measurement equip ment is that the time between stopping the mixing and recording it photographically is reduced to $2 \mathrm{~s}$ and the foam is not damaged during transport to the photographic cell.

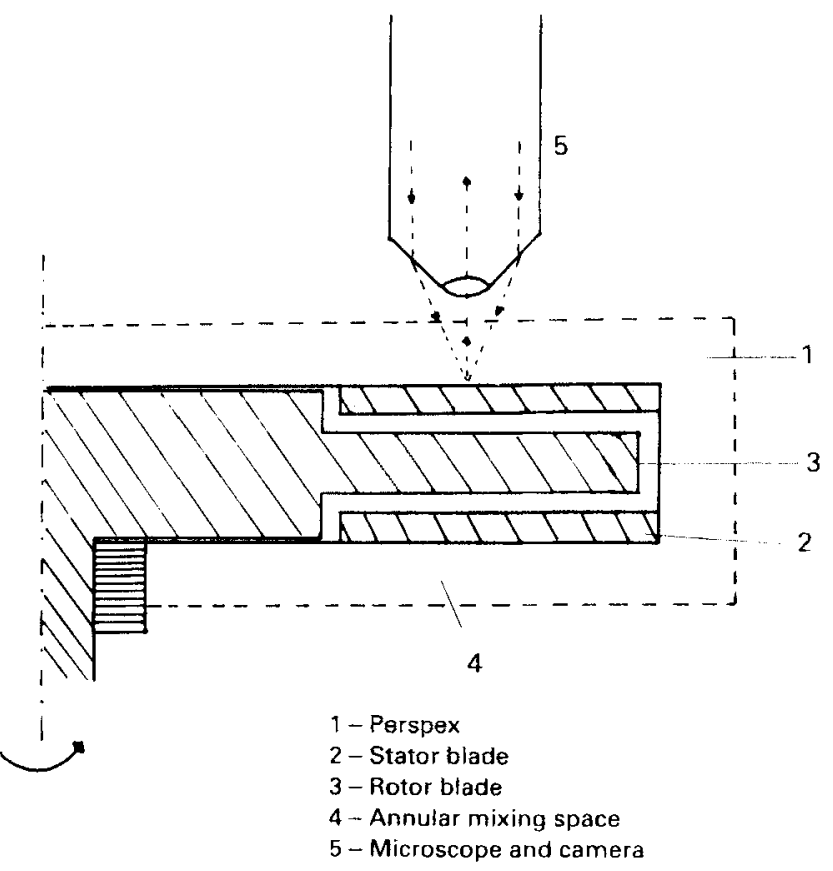

Figure 3 - Rotor mixer unit

The energy supplied to the mixer can be measured in the equipment. The torque of the rotor shaft between the mixer head and the motor can be measured. The energy supplied can be calculated by Eqn 11:

$$
\mathrm{Q}_{w}=2 \pi N M
$$

For the experiments a solution is used containing $1.5 \%$ Diphasol EA, 1\% Diphasol VA (both CGY) and $0.1 \%$ acetic acid in water with varying concentrations of starch ether as thickener; Table 2 gives a summary of the concentrations of starch ether used. The viscosity of the solutions was measured with a coaxial cylinder viscometer with sheer rates from 50 to $500 \mathrm{~s}^{-1}$. A power law was used to correlate the shear stress as a function of shear rate (Eqn 12):

$$
T=\mu D_{r}^{n}
$$

Extrapolation of the viscosity to higher shear rates was necessary in order to calculate the apparent viscosity in the mixer. The surface tension of the solution without thickeners was measured as $0.03 \mathrm{~N} / \mathrm{m}$. For the solutions with CMC thickener a value of $0.03 \mathrm{~N} / \mathrm{m}$ was used, assuming that the surface tension was unaltered by the thickeners. 
Viscosities of the systems used

\begin{tabular}{rcccc}
\hline System & $\begin{array}{c}\text { Arnount of } \\
\text { thickener } \\
(\text { wt } \%)\end{array}$ & $\begin{array}{c}\text { Foam } \\
\text { density } \\
\left(\mathrm{kg} / \mathrm{m}^{3}\right)\end{array}$ & $\begin{array}{c}\mu \\
\left(\mathrm{Pa} \mathrm{s}^{n}\right)\end{array}$ & $n$ \\
\hline 1 & 0 & 1000 & 0.001 & 1 \\
2 & 0 & 800 & $\sim 0.001$ & $\sim 1$ \\
3 & 1.4 & 1000 & 0.114 & 0.8 \\
4 & 2.8 & 1000 & 1.21 & 0.63 \\
5 & 2.8 & 1000 & 1.14 & 0.69 \\
6 & 2.8 & 720 & 3.23 & 0.55 \\
7 & 2.8 & 550 & 5.55 & 0.5 \\
8 & 2.8 & 320 & 8.54 & 0.48 \\
9 & 6.0 & 1000 & 34.5 & 0.43 \\
10 & 6.0 & 790 & 46.2 & 0.4 \\
11 & Glycerine & & 1.16 & 1 \\
12 & 3.0 & 450 & 7.6 & 0.55 \\
13 & 3.0 & 655 & 2.9 & 0.65 \\
14 & 4.5 & 550 & 25.3 & 0.48 \\
\hline
\end{tabular}

\section{Measuring the bubble size distribution}

The bubble size distribution was recorded photographically in the plane next to the wetted window of the measurement cell. The size of the photographically recorded bubbles was measured and divided into about 15 classes.

The number of bubbles measured in every experiment was about 250. Based on these classifications the experiments were evaluated. The distribution was characterised by the mean bubble size and the standard deviation of the bubble size. The mean bubble size to the $j$ th and the ith moment is defined by Eqn 13 :

$$
d(j, i)=\left(\frac{\int_{0}^{\infty} d^{i} f(d) \mathrm{d} d}{\int_{0}^{\infty} d^{i} f(d) \mathrm{d} d}\right)^{1 /(j-i)}
$$

Depending on the use of the mean bubble size, special moments may be preferred. We shall use the mean diameter with the moments $j=1$ and $i=0[d(1,0)]$ :

$$
d(1,0)=\frac{\int_{0}^{\infty} d f(d) \mathrm{d} d}{\int_{0}^{\infty} \mathrm{f}(d) \mathrm{d} d}
$$

The following errors in bubble size measurements caused a discrepancy between the real bubble size distribution and the measured distribution next to a transparent plane.

(a) De Vries [2] showed that larger bubbles will be detected relatively often in comparison with smaller bubbles because a larger bubble is a larger target. He used the correction factor given in Eqn 15:

$$
\mathrm{f}^{\prime}(r)=\int_{0}^{\infty}(\mathrm{f}(r) / r) \mathrm{d} r \cdot \frac{\mathrm{f}(r)}{r}
$$

The mean bubble diameter of the uncorrected distribution function with the moments $j$ and $i$ is equal to the mean bubble diameter of the corrected distribution function with the moments $j-1$ and $i-1$. The uncorrected $d(0,-1)$ is equal to $d(1,0)$.

(b) Cheng and Lemlich [13] showed that the bubbles are actually larger than they appear to be when photo- graphed at a transparent surface (Figure 4). Depending of the size of the bubble a definite error in the bubble diameter will be made.

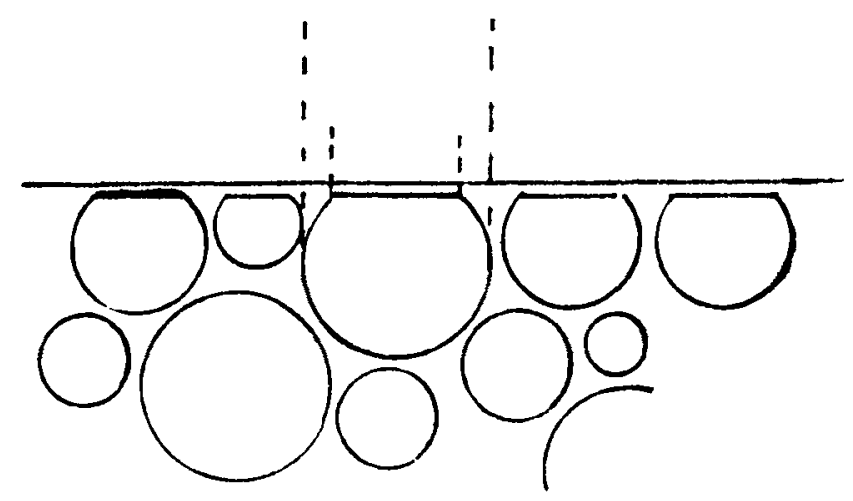

Figure 4-Observation error

(c) Cheng and Lemlich [13] have measured a difference in the bubble size distribution in the foam and at the transparent surface. Due to segregation smaller bubbles are recorded with relatively greater frequency than larger bubbles.

(d) At low blow ratios (less than unity) the transparent plane in the measurement cell is not completely filled with bubbles (Figure 5). Bubbles at a greater distance from the transparent plane are visible. Depending of the depth of field of the photographic unit, bubbles can be recorded at a lesser or greater distance. Larger bubbles will be visible at a greater distance than smaller bubbles. Koetsier [14] found that the depth at which the bubbles are visible varies linearly with the bubble diameter.

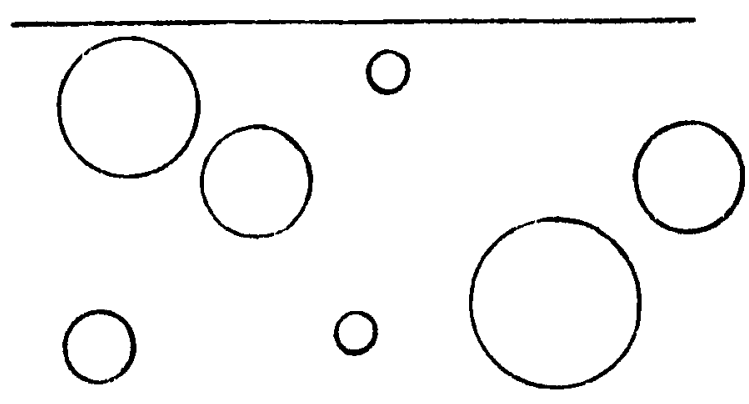

Figure 5 - Gas emulsions with low blow ratio

The de Vries correction factor was used to calculate the real bubble size distribution in the foam from the experimental results. Other errors in the bubble size measurement were not taken into account. The bubble size distribution is characterised by a mean diameter $d(1,0)$, and the standard deviation, $\sigma$, in the measured and corrected distribution. The parameters $d+$ and $d-$ were used in order to trace an upper and a lower diameter to characterise the deviation in the distribution function $(d+=d(1,0)+\sigma$ and $d-=d(1,0)-\sigma)$.

\section{EXPERIMENTAL RESULTS}

Energy dissipation in the foam mixer

The energy dissipation in the mixer is an important quantity for determining the shear stresses, which control 
the bubble splitting. The energy dissipation in a foam mixer with a given geometry is determined by two dimensionless numbers, the Reynolds number and the Newton number (Eqns 16 and 17):

$$
\begin{gathered}
(N e)=\frac{Q_{w}}{e^{5} \rho N^{3}} \\
(R e)=\frac{\rho N e^{2}}{\mu}
\end{gathered}
$$

The relationship between the Newton number and the Reynolds number is given by Eqn 18 the NewtonReynolds expression):

$$
(N e)=f(R e)
$$

The Newton-Reynolds expression can be determined by power consumption measurements in the mixer. Metzer and Otto [15] have recommended the following procedure to determine the expression for nonNewtonian fluids. They give a procedure in seven steps.

1. Determine the Newton-Reynolds expression for a Newtonian fluid.

2. Determine in the laminar field the Newton numbers for a non-Newtonian fluid (pseudoplastics).

3. Determine the Reynolds numbers corresponding to the Newton numbers found in step 2 with the Newton-Reynolds expression of step 1 .

4. The calculated Reynolds numbers give the apparent viscosities of the pseudoplastics used. The relation between the apparent viscosity in the mixer and the number of revolutions can be determined $\left(U_{2} \approx N^{(n-1)}\right)$.

5. Determine the viscosity of the pseudoplastic as a function of the shear rate $\left(U_{1}=\mu D_{\mathrm{r}}^{(n-1)}\right)$.

6. Put $U_{1}=U_{2}$ and determine the relationship $D_{\mathrm{r}}=k N$. The mixer property $k$ represents the relationship between the number of revolutions per second of the mixer rotor and the mean shear rate in the mixer.

7. The modified Reynolds number for fluids with a power law rheology can be written as: $(R e)=\rho N^{(2-n)} e^{2} / \mu k^{(n-1)}$.

The Newton-Reynolds expression of a rotor-stator mixer was determined with the procedure described. The Newton and Reynolds numbers used were adapted to the relevant geometry of the mixer as specified by Eqns 19 and 20:

$$
\begin{aligned}
& (N e)=\frac{Q_{w}}{e^{4} \rho / h N^{3}} \\
& (R e)=\frac{\rho N^{(2-h)} e p}{\mu k^{(n-1)}}
\end{aligned}
$$

The dimensions of the mixer used were $e=45 \mathrm{~mm}$, $p=1 \mathrm{~mm}$ and $l=17 \mathrm{~mm}$. The viscosity of each solution and of the foam was measured in the shear rate range from 50 to $500 \mathrm{~s}^{-1}$. The results are represented in Table 2 . Extrapolation of the viscosities to the high shear rates in the mixer using a power law function gave good results. The value of the mixer constant $k$ is about 100 . The relationship between the number of revolutions per second and the mean value of the shear rate in the laminar flow is given by Eqn 21:

$$
D_{\mathrm{r}}=100 N
$$

For the density of the fluid appearing in the Newton and Reynolds numbers, the mean foam density was used $\left(\rho_{\mathrm{s}}=\rho_{\mathrm{l}} /\left(1+B_{\mathrm{m}}\right)\right)$. In the turbulent flow field the energy consumption was found to be linearly related to mean foam density at a given rotational speed. Viscosities of foams of solutions without thickener were not measured, therefore these Newton numbers are not represented in Figure 6. The Newton numbers of the mixer for these foams were all about 10 . In the Newton-Reynolds expression two areas can be distinguished. At lower Reynolds numbers $(<5.7)$ the Newton-Reynolds expression can be described by Eqn 22 and for the higher Reynolds numbers ( $>5.7$ ) Eqn 23 can be used. Between a Reynolds number of 5 and 10 the flow properties in the mixer change from laminar to turbulent flow.

$$
\begin{gathered}
N e=57 /(R e) \\
N e=10
\end{gathered}
$$

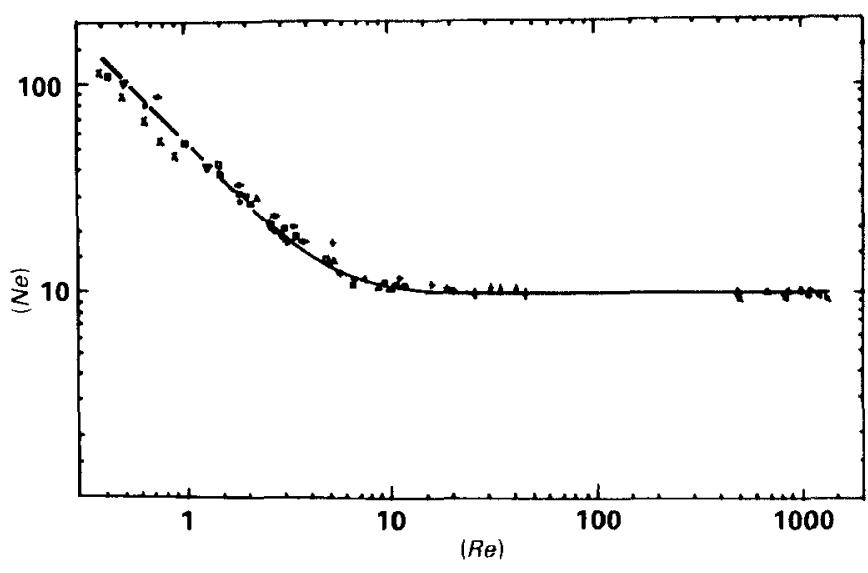

Figure 6 - Newton-Reynolds expression for foam mixer

\section{Mean bubble size and residence time}

The foam was produced in an annular mixing space containing rotors and stators. The first section of the mixer was used to disperse the gas in the foam phase, and the blow ratio of the foam phase decreased in this section until all the gas was dispersed [1]. In the second section of the mixer only the degree of dispersion characterised by the bubble size distribution changed.

The mean bubble size is represented as a function of the residence time of the foam in the mixer in Figure 7 for three different blow ratios. The unthickened solution was foamed and the bubble size distribution was measured. The rotational speed of the mixer was $20 \mathrm{rev} . / \mathrm{s}$. At short residence times the mean bubble size was highly dependent on the residence time, while at longer residence times an equilibrium mean bubble size was obtained. To produce a foam with a lower density, a larger residence time was needed to obtain the equilibrium mean bubble size. One of the reasons is that more time is required for pre-mixing. Relatively longer resi dence times were used when measuring bubble size distribution as a function of the mixing properties, in order to obtain an equilibrium value.

\section{Bubble size distribution as a function of the shear rate in the mixer}

Bubble size distributions were measured in both shear fields and in the mixer. 


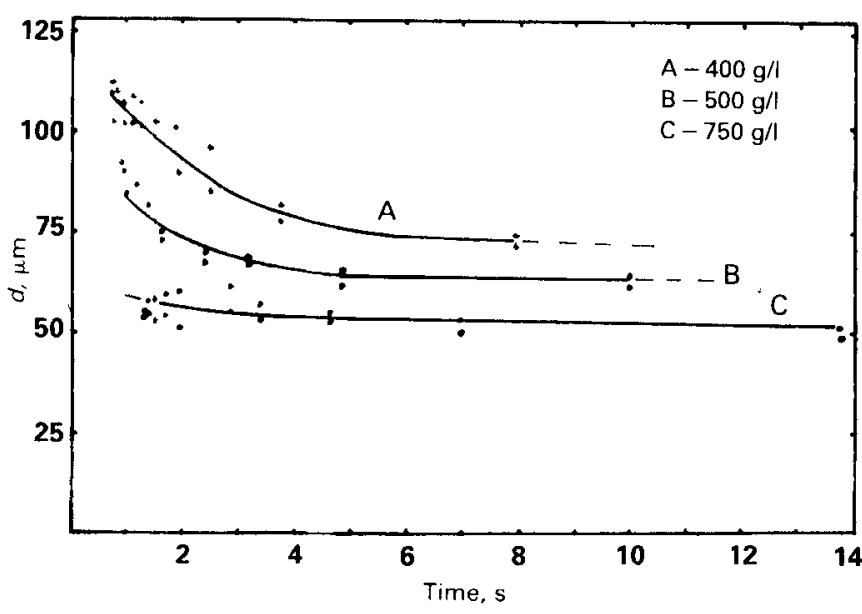

Figure 7 - Mean bubble size as a function of residence time

\section{Turbulent flow field}

Measurements were carried out with foam densities from 250 to $900 \mathrm{~g} / 1$ in the mixer with rotational speeds in the range 18 to $43 \mathrm{rev} . / \mathrm{s}$. The mixing time in every experiment was 10 s. Figure 8 shows the mean bubble size as a function of the parameter $N_{\mathrm{t}}$, and Eqn 24 represents the relationship between the mean bubble diameter, $d(1,0)$, and the parameter $N_{\mathrm{t}}$ :

$$
d(1,0)=28700 N_{\mathrm{t}}^{-1.03}
$$

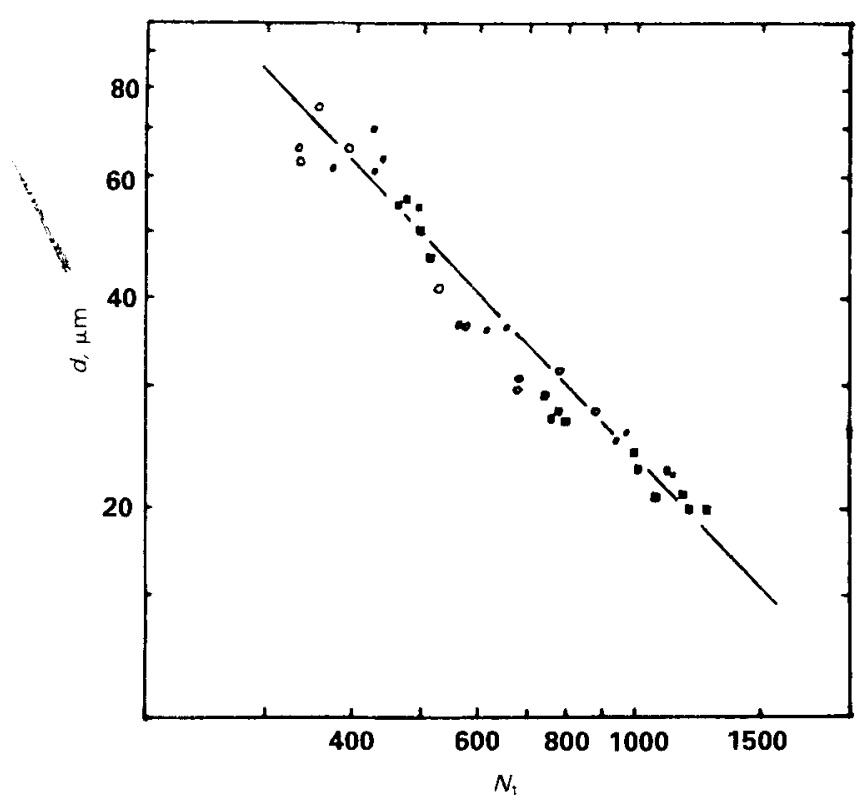

Figure 8 -Plot of $\mathrm{d}(1,0)$ versus $\mathrm{N}_{t}$

Figure 9 shows the standard deviation of the distribution as a function of the mean bubble diameter; this relation is also defined by Eqn 25 . Clearly at smaller bubble diameters the standard deviation is relatively small:

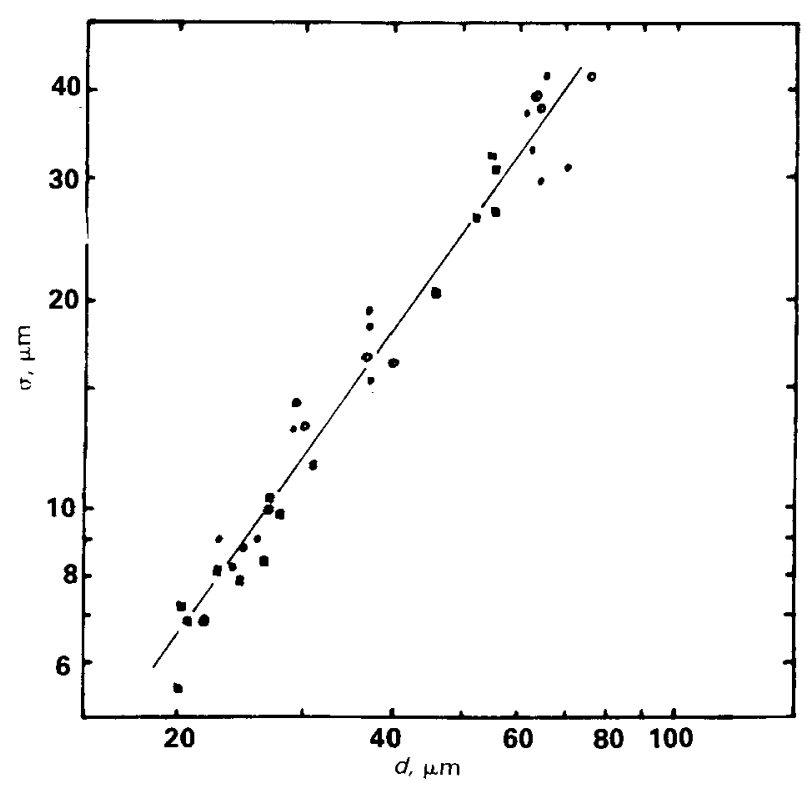

Figure 9 - Plot of $\sigma$ versus $\mathrm{d}(1,0)$

The variance of the distribution can be represented in terms of $d+$ and $d$ - as a function of $N_{\mathrm{t}}$, according to Eqns 26 and 27 (in $\mu \mathrm{m}$ ):

$$
\begin{gathered}
d+=124000 N_{\mathrm{t}}^{-1.2} \\
d-=1130 N_{\mathrm{t}}^{-0.62}
\end{gathered}
$$

Thus the bubble diameter $d+$ shows a behaviour similar to that of the maximum stable bubble diameter as given by Eqn 10, in combination with Eqn 23. Thus bubble diameter $d+$ may be described using the concept of a critical Weber number.

The number of bubbles counted per experiment was too small for the determination of an accurate distribution. Therefore the experiments were divided into four groups, each within a certain range of the parameter $N_{\mathrm{t}}$. The results of one group of experiments were averaged, after normalising bubble diameters with respect to the mean. Every experiment was based on the diameters of about 2000 bubbles. Figure 10 represents the measured cumulative distributions. The change in the shape of the distribution is very clear.

\section{Laminar flow field}

The solutions used and foam densities used for investigations in the laminar flow field are given in Table 2 (systems 12-14). The number of revolutions per second was in the range 11 to 44 . Figure 11 represents the mean bubble diameter as a function of the mean shear stress in the mixer $\left[T=\mu(100 N)^{n}\right]$. This relationship is also given by Eqn 18 (in $\mu \mathrm{m}$ ):

$$
d(1,0)=3.1 T^{-0.81}
$$

Figure 12 represents the standard deviation in the bubble size distribution as a function of the mean bubble diameter; Eqn 29 gives the standard deviation as a function of the mean bubble diameter:

$$
\boldsymbol{\sigma}=0.27[d(1,0)]^{1.22}
$$



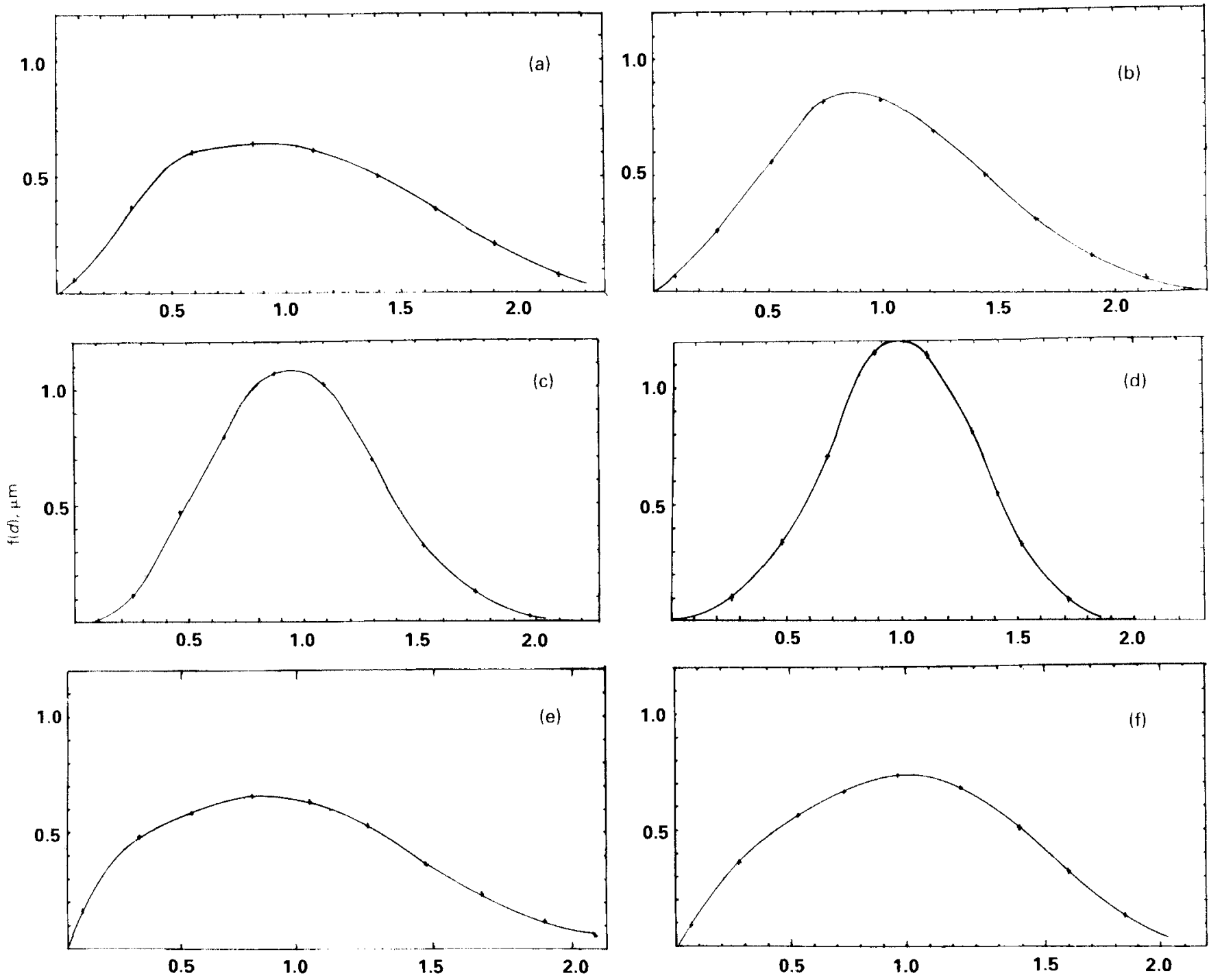

(a) $300: N_{1} \cdot 500$
(c) $700<N_{1}<950$
(e) $380 \div T>700$

(b) $500 N_{1} \div 700$

(d) $950 N_{1}=1200$

(f) $700 T 1000$

Figure 10 - Measured distribution as a function of diameter for four regions of $\mathrm{N}_{t}$ in the turbulent flow field $(\mathrm{a}-\mathrm{d})$ and two regions of $\mathrm{T}$ in the laminar flow field ( $e$ and $f$ )

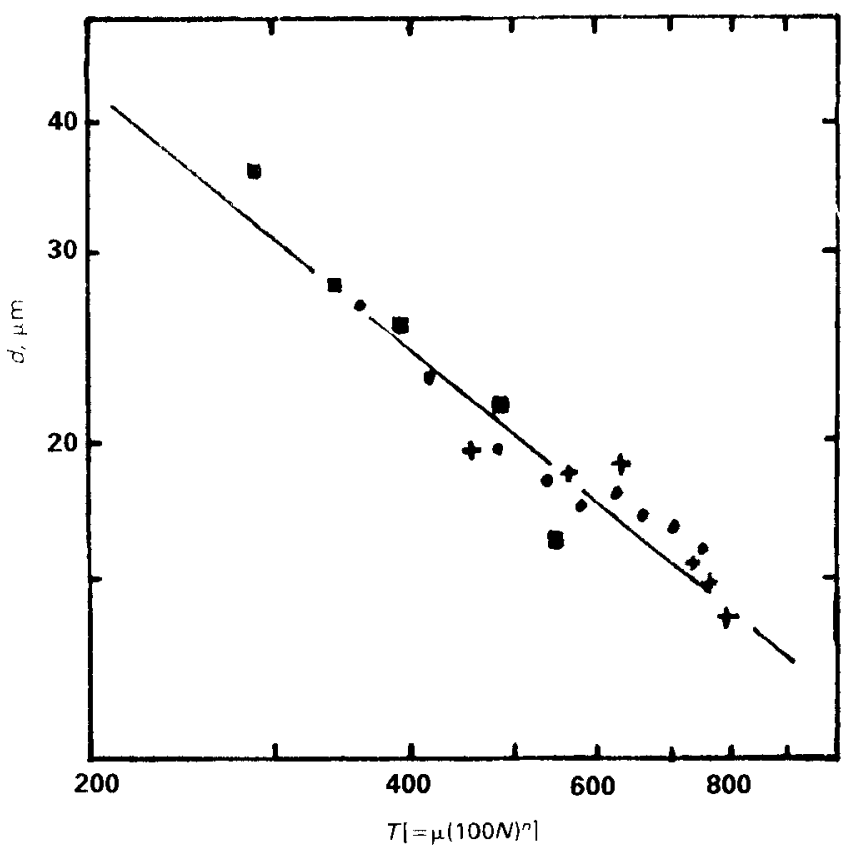

Figure 11 - Plot of $\mathrm{d}(1,0)$ versus $\mathrm{T}$

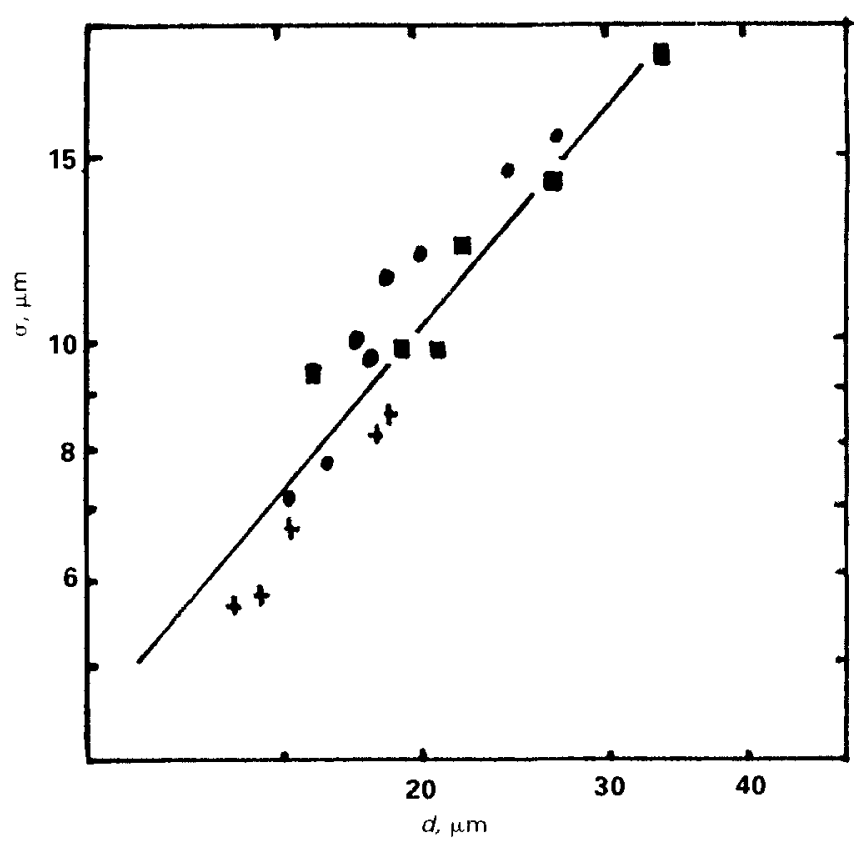

Figure 12 - Plot of o versus d 
Changes in standard deviation in the bubble size distribution were not linearly related to mean bubble diameter. As in the experiments carried out under turbulent flow conditions, the experiments carried out in the laminar flow region were also evaluated in terms of $d+$ and $d-$. Eqns 30 and 31 give the values of $d+$ and $d-$ as a function of the mean shear rate in the mixer (in $\mu \mathrm{m}$ ):

$$
\begin{gathered}
d+=8.3 T^{-0.9} \\
d-=0.18 T^{-0.48}
\end{gathered}
$$

If $d+$ can be described in terms of a critical Weber number, the exponent of $T$ in Eqn 30 could be expected to be -1 . In reality the exponent of $T$ is a somewhat lower. However, taking into account the spread in the measurements, the difference between theory and experiment is not excessive.

In order to determine a complete distribution, cumulative distribution functions are calculated after dividing the experiments into two groups. The distribution functions are represented in Figure 10.

\section{EVALUATION}

Bubble diameter in a foam can be correlated with the mixing properties in the turbulent as well as in the laminar field. In the transitional zone (at low rates of turbulent flow) no experiments were carried out. In this area the shear causing bubble splitting changes from inertial to viscous shear. In the laminar flow field the bubble diameter $d+$ can be characterised by the Weber number, using the mean shear in the mixer (Eqn 32):

$$
(W e)_{c}=\frac{T d+}{\gamma} \approx 0.6
$$

The experiments gave no indication about the validity of using the mean shear rate or maximum shear rate in the mixer. When a rotor passes a stator pen, the shear rate attains a maximum value. The relationship for the mean shear rate in the mixer measured by energy consumption measurements is given by Eqn 21. The maximum shear rate might be approximated by assuming an ideal shear flow at the moment a rotor passes a stator pen. The relationship between the shear rate and the number of revolutions is then given approximately by Eqn 33:

$$
D_{\mathrm{r}, \max }=160 \mathrm{~N}
$$

The difference in the maximum and the mean shear rates is therefore a factor of about 1.6. This leads to a difference in the maximum and mean shear stress of about $20 \%$ for a pseudoplast, e.g. a foam with an $n$ value of 0.4. In the turbulent flow field the bubble diameter $d+$ can also be correlated with the critical Weber number (Eqn 34):

$$
(W e)_{\mathrm{c}}=\frac{T d+}{\gamma} \approx 0.23
$$

The turbulent shear stress can be calculated by means of Eqns 10 and 23, the limiting condition for using the turbulence theory for calculating the shear stress being $L_{1} \gg d \gg L_{\mathrm{s}}$. When $L_{1}$ was the distance between the rotor and the stator the ratio $L_{1} / e$ was larger than 10 in all the experiments. The scale of the smallest energy dissipating eddies can be calculated by Eqn 7. For instance, a foam density of $250 \mathrm{~g} / \mathrm{l}$ in the mixer rotating at $10 \mathrm{rev} . / \mathrm{s}$ and with a viscosity of the liquid of $1 \mathrm{cp}$ leads to a smallest eddy of about $3 \mu \mathrm{m}$.

The question of the disturbance of the turbulent character by the large number of bubbles in the field has not been answered in this investigation, but experimentally no significant difference was found when increasing the amount of gas in the foam from 10 to $75 \%$.

In order to calculate the shear stresses in the mixer the foam can be considered as a homogeneous medium with its own density and viscosity. The properties of the separated phases are of minor importance, compared with the properties of the dispersed phase. The change in bubble size distribution of the foam as a function of foam density was primarily determined by the changing shear stresses in the mixer. The influence of a higher rate of coalescence appeared to be of minor importance, as was suspected. The foam was apparently quite effectively stabilised by the surface-active agents used.

It is striking that in the laminar and turbulent regions the variance in the bubble size distribution did not vary linearly with the mean bubble diameter. A distribution with a large mean bubble diameter also had a relatively large standard deviation.

\section{CONCLUSIONS}

Several parameters have been investigated that determine the bubble size distribution of a foam generated in a rotor-stator mixer. For practical purposes we may conclude that, when a given liquid system is mixed to a foam of a given blow ratio in a rotor-stator mixer, the only parameter that can be used to change the bubble size distribution is the rotational speed of the mixer. The residence time is a less important parameter because the bubble size distribution reaches an equilibrium within several seconds and shorter residence times are less attractive because of the large variance obtained in the distribution. Another point to take into account is that while very small bubble sizes may be generated in the mixer, a long residence time between generation and application can cause a significant enlargement of the bubbles due to gas diffusion.

In detail the conclusions can be summarised as follows.

1. The stationary bubble size distribution in the mixing system studied is primarily determined by the shear stresses in the mixer.

2. A laminar and a turbulent flow field can be determined in the mixer by energy consumption measurements.

3. When correlating the bubble size distribution in terms of the mean bubble size $[d(1,0)]$ and the standard deviation $(\sigma)$ as a function of the mixing parameters, the bubble size $d+[=d(1,0)+\sigma]$ can be correlated in terms of a critical Weber number.

4. The standard deviation in the bubble size distribution function does not vary linearly with the mean bubble size. A distribution with a larger mean diameter has relatively greater standard deviation.

* $\quad * \quad$ *

These investigations were supported (in part) by the Netherlands Foundation for the Technical Sciences 
(STW), Technical Science Branch of the Netherlands Organisation for the Advancement of Pure Research (ZWO)

\section{REFERENCES}

1. A B T Kroezen and J Groot Wassink, J.S.D.C., 102 (1986) 397

2. A J de Vries, PhD thesis (Delft, 1957).

3. J J Bikerman, 'Foams' (Berlin: Springer, 1973)

4 A Ranadine and R Lemlich, J. Colloid Interfacial Sci, 70 (2) (1979) 392

5. G I Taylor, Proc. Royal Soc., A146 (1934) 501

6. CI Taylor, Proc. Royal Soc, A136 (1932) 41

7. FD Rumscheid and G Mason, J. Colloid and Interfacial Sci., 16 (1961) 238

P Kiefer, PhD thesis (Karlsruhe, 1977).

9 G K Batchelor, Proc. Cambridge Phil. Soc. 47 (1951) 359

10 R K Baipai, D Ramkrihna and A Prokop, Chem. Eng. Sci., 31 (1976) 913

11 A Hsia and I. Taularides, Chem. Eng. t., 20 (1980) 225.

12 M Konno et al., J. Chem. Eng. Japan, 13 (1) (1980) 67.

13 A Cheng and R L.emlich. Ind. Eng. Chem., 22 (1983) 105

14 W Koetsier, PhD thesis (Enschede, 1973).

15 A B Metzer and R E Offo, AIChE J., 3 (1957) 3.

16 B Gall Or, International J. Heat Mass Transfer, 11 (1968) 551.

17 W van Heuven, PhD thesis (Delft, 1969).

18 A Pawelczyk, Inz. Chem. Proc., 2 (1980) 267

\section{LIST OF SYMBOLS}

Symbol Description

$a, b \quad$ Bubble dimensions

$B, B_{\text {o, }}$ Blow ratio, at atmospheric

$B_{\mathrm{m}} \mid$ pressure, at mixer pressure

$c_{1} \quad$ Constant in Eqn 8

d Bubble diameter

$d(i, j) \quad$ Mean diameter with moment $i, j \mathrm{~m}$

$d_{\mathrm{o}}, d_{\mathrm{m}}$ Diameter of the bubble at $\mathrm{m}$ atmospheric pressure, at mixer pressure

$d+, d-d(1,0)+\sigma, d(1,0)-\sigma$

$D_{\mathrm{r}} \quad$ Shear rate

(D) Deformation number of the bubble

$e \quad$ Mixer diameter

Unit

m

$f(r), f(d) \quad$ Distribution function $h \quad$ Number of rotor-stator units of the mixer

$j, i \quad$ Moments of the mean bubble

size

$k \quad$ Mixer constants

$L_{\mathrm{s}}, L_{\mathrm{l}} \quad$ Scale of eddies in the turbulent $\mathrm{m}$ field

I Diameter of the annular space inm the mixer

$M \quad$ Moment of the rotor shaft $\quad \mathrm{N} \mathrm{m}$

$n \quad$ Rheological constant (defined by

Eqn 12)

$N \quad$ Rotational speed (rev./s) $\quad \mathrm{s}^{-1}$

$N_{\mathrm{t}} \quad \rho_{\mathrm{s}}{ }^{1 / 2} \mathrm{~N}$

$(\mathrm{Ne}) \quad$ Newton number

$P, P_{\mathrm{o}}, P_{\mathrm{m}}$ Pressure, atmospheric, in the $\mathrm{N} / \mathrm{m}^{2}$ mixer

$Q_{w} / V \quad$ Power dissipation per unit $\quad \mathrm{N} /\left(\mathrm{m}^{2} \mathrm{~s}\right)$ volume

$p \quad$ Rotor-stator distance in the $m$ mixer

$r, r_{1}, r_{2} \quad$ Bubble radius $\mathrm{m}$

(Re) Reynolds number

$T \quad$ Shear stress

$v \quad$ Velocity

V Volume

(We) Weber number

$(W e)_{\mathrm{c}} \quad$ Critical Weber number

$\gamma \quad$ Surface tension N/m

$\lambda \quad \mu_{i} / \mu_{a}$

$\mu \quad$ Rheological constant (defined Pa s${ }^{n}$ by Eqn 12)

$\mu_{\mathrm{a}} \quad$ Viscosity of continuous phase $\mathrm{Pa} \mathrm{s}$

$\mu_{i} \quad$ Viscosity of dispersed phase $\quad \mathrm{Pas}$

$\rho$ Density $\mathrm{kg} / \mathrm{m}^{3}$

$\rho_{l} \quad$ Density of liquid $\mathrm{kg} / \mathrm{m}^{3}$

$\rho_{\mathrm{s}} \quad$ Mean density of foam $\left(\rho_{\mathrm{l}} /(1+B)\right) \mathrm{kg} / \mathrm{m}^{3}$

$\sigma \quad$ Standard deviation

\section{YEARS AGO}

Extract from report on 'the Combrook explosion', originally published in The Times, Journal, November 1887

On June 22nd, the first intimation of anything unusual was the outbreak of a fire about seven or eight minutes before noon, which spread with great rapidity. After the lapse of five or six minutes there was an explosion of a moderate character, which caused no serious damage, but after an interval of not more than a minute a second explosion of a most tremendous and destructive character took place. The works themselves were practically annihilated, machinery and apparatus distorted and destroyed, and the air filled with dense clouds of smoke and acrid picric vapours and dust, with masses of projected débris, much of the dust and débris being on fire, and thus being the means of carrying it to other buildings. The force of the explosion was felt within a radius of two miles, and the report was distinctly heard a distance of 20 miles. It has been estimated that the force exerted by the explosion was equal to that produced by a charge of $500 \mathrm{lb}$ of gun cotton.

In the opinion of Col. Majendie (HM chief inspector of explosives) the explosion was the result of the 'negligent manner' in which certain substances as litharge, nitrate of lead. or nitrate of strontium were stored. 